\title{
EL CRECIENTE USO DE LAS NUEVAS TECNOLOGÍAS EN ADULTOS MAYORES
}

\author{
Alejandro Klein
}

resumen

Teniendo en cuenta que las computadoras se han vuelto sustantivamente parte integral de la vida de los adultos mayores, el estudio del uso de las mismas por esta población se está volviendo de forma incrementada un sustancial campo de estudio. El objetivo del estudio es una revisión bibliográfica para comprender mejor la relación de las nuevas tecnologías con la población de adultos mayores. Una de las consecuencias que se desprende es que para animar y facilitar el uso de las nuevas tecnologias es necesario comprender cuáles son las implicaciones de estas diferencias en el uso de las nuevas tecnologías y las tradiciones culturales. La metodología utilizada es de revisión bibliográfica en la que se ha dado énfasis tanto a la evolución de las perspectivas académicas sobre el tema, como los resultados obtenidos entre los años 1970 a 2016. Hay que señalar que todas las investigaciones consultadas insisten en el papel cada vez más protagónico de las nuevas tecnología de información en la vida de la tercera edad, y que lejos de suponer que los mismos tendrían un rechazo a las mismas, se percibe un uso operativo y eficaz de las nuevas tecnologías.

1 Graduado en Psicología. Doctor en Trabajo Social (Universidad Federal de Rio de Janeiro). Profesor titular de la Universidad de Guanajuato. E-mail: alejandroklein@hotmail.com. 


\section{Introducción}

Estimase que hasta el año 2050, el 21.8\% de la población mundial será de adultos mayores (UNITED NATIONS, 2008). En los estudios de los años 90' se estimaba que el grupo de individuos de 75 años y más constituían el grupo de adultos mayores de mayor crecimiento (LAWHORN et al., 1996; SCHNEIDER, 1991). Sin embargo las últimas investigaciones señalan que el grupo de tercera edad que mayor crece es el de los centenarios (LEESON, 2009; 2013; UNITED NATIONS, 2008).

La habilidad para el uso de herramientas de información y comunicación es considerado cada vez más como un requisito esencial para estos adultos mayores en lo que se denomina "era tecnológica" (WILLS, 1999; BLASCO; MELÉNDEZ, 2006). Se habla en este sentido de alfabetización digital como el esfuerzo que la población debe hacer y especialmente los adultos mayores para reformular sus estructuras cognitivas y de alfabetización desde las nuevas estrategias de pensamiento, acción y coordinación psicomotora que implican las nuevas tecnologías (CERDA, 2005). Otros beneficios se relacionan con el mejor manejo de sus problemas, información de salud, mejores interacciones, asistencia en el trabajo, planificación de viajes, compras y manejo financiero, ayudando a mejorar vínculos y redes sociales y culturales (ADLER, 1996; CODY et al., 1999; LOGES; JUNG, 2001; WHITE et al., 1999; IRIZARRY; DOWNING, 1997).

Se ha hablado inclusive de "silver surfers" para describir esta situación (BRAYFIELD, 2000; CODY et al., 1999; COPPS, 2000). La noción de "silver surfer" implica el entendido de que los adultos mayores se benefician de los TIC de distintas maneras, una de las cuales es "bridge the generation gap" (BURDICK, 2001).

Sin embargo este punto de vista ya no es mantenido unánimemente, priorizando actualmente aspectos que parecen muchos más relevantes en torno a la promoción de ciudadanía, empowerment y agenciamiento, destacándose la mayor capacidad de participación política y social de los adultos mayores (BERNARD; PHILLIPS, 2000; KLEIN; AVILA-EGGLETON, 2011). A pesar de estos aspectos positivos se indica que existe aún un porcentaje de adultos mayores excluidos de las nuevas tecnologías (HANLEY, 2002; MADDEN; SAVAGE, 2000; TEO, 2001) especialmente por diferencias sociales y económicas que afectan a los adultos mayores de menos recursos económicos lo que genera 
preocupación (JARA, 2008), y implica la necesidad de orientar políticas públicas que apoyen a compensar estas desigualdades sociales (SANTANA, 2015; MARQUINE; SANTANA, 2016; BILBAO-OSORIO; DUTTA; LANVIN, 2014).

\section{Hipótesis general}

El presente estudio presenta la hipótesis de que los adultos mayores se encuentran insertos en una sociedad de tecnología y por lo tanto en mayor o menor grado no pueden desentenderse de la misma. Podríamos decir que existen tecnologías "cotidianas" (teléfono, celulares) y otras más "sofisticadas" como el uso de las computadores y aparatos anexos a la misma que plantean diferentes retos y desafíos. Como se advertirá en la bibliografía consultada (VAN DER WARDT; BANDELOW; HOGERVORST, 2012; CHUTTUR, 2009; EZER; FISK; ROGERS, 2009) se entiende que a mayor uso de las tecnologías "sofisticadas" existe un mayor nivel de satisfacción vital, experimentando los adultos mayores más sentimiento de autosuficiencia. La bibliografía referida (VAN DER WARDT; BANDELOW; HOGERVORST, 2012; CHUTTUR, 2009; EZER; FISK; ROGERS, 2009) también indica que existen diferencias de género al respecto, sintiéndose las mujeres con mayor ansiedad frente al uso de computadoras. En este sentido su área vital parece restringirse más a la tecnología "cotidiana" que a la "sofisticada".

Sin embargo estos dos puntos merecen ser revisados. Aunque muchos autores (KUBIK, 2009; OPPENAUER, 2009; BOUMA et al., 2007) consideran que el uso de computadoras y las derivaciones a partir de ellas (como chats, skype, youtube, otros) aumentan la calidad de vida con menos sentimientos de soledad y aislamiento, consideramos por otro lado que existe cierto "forzamiento" y presión social para que el adulto mayor aprenda y conozca estas nuevas tecnologías. Tampoco es claro que el ser "hombre" o "mujer" facilite a priori el uso de estas tecnologías. Asimismo es necesario indicar que la realidad angloparlante o europea no es la misma que la de otros países, quienes se encuentran en un punto de menor difusión tecnológica con consiguientes problemas de déficit en la llamada alfabetización digital.

\section{Referencial teórico}

En esta sección serán tratados diferentes aspectos que problematizan, tanto como dejan el debate abierto, acerca del uso de las nuevas tecnologías (sus problemáticas, desafíos e incidencias) en la población de adultos mayores. 
Los adultos mayores están aprendiendo cada vez más a interactuar con las distintas formas de tecnología, entendiéndose que se trata de un logro que mantiene su independencia y reduce su necesidad de ser cuidados. En términos de tratamiento médico provee la posibilidad de controlar condiciones médicas a distancia, reduciendo el uso de medicinas y malos procedimientos en tratamientos de enfermedad. Asimismo implica una reducción significativa de los costos de salud (FIELD et al., 2005).

Asimismo, el uso de estas tecnologías favorece en algunos adultos mayores la preferencia por permanecer en sus propias casas, viviendo de forma independiente (US CENSUS BUREAU, 2000; AARP, 1996). La tecnología permite que la mayoría de las actividades ocurran dentro de la "protección" del hogar (BALTES et al., 1999; AARP, 2008). Esto podría relacionarse a la percepción social de que las nuevas tecnologías generan más beneficios que costos (MELENHORST, ROGERS; BOUWHUIS, 2006).

A pesar de eso el porcentaje de adultos mayores que usan tecnología, por ejemplo en Estados Unidos, es aún bajo: 25\% en la población de 65 años o más, $56 \%$ en la población entre 55 y 64 años y un $68 \%$ en la población entre 25 y 54 años (US CENSUS, 2003). Sin embargo, las últimas investigaciones señalan que este número se incrementa incesantemente en la población de 65 años y más (SANTANA, 2015, MARQUINE; SANTANA, 2016; BILBAO-OSORIO; DUTTA; LANVIN, 2014).

Estos datos parecen indicar, como conclusión preliminar, que el porcentaje de adultos mayores que comienza a explorar el mundo de la tecnología es cada vez mayor, especialmente en el uso de internet que es cada vez más incorporado a la vida cotidiana como un elemento habitual y no extraño (HART; HALCOMB; CHAPARRO, 2008; LOGES; JUNG, 2001; GREEN; MCADAMS, 2003). De esta manera el grupo de tercera edad utiliza cada vez más servicios, compras, transacciones bancarias y planificación de actividades de placer vía online sin que esto le resulte conflictivo o angustiante (VUORI; HOLMLUND-RYTKÖNEN, 2005).

\subsection{Factores que predicen el uso de tecnología}

Es importante poder predecir cuáles son los factores que facilitan o dificultan el uso de la tecnología. En ese sentido se propone al TAM (en inglés: Technology Acceptance Model - TAM) como un modelo teórico para comprender 
la aceptación de las tecnologías en la vida cotidiana (percepción de facilidad de la misma y su utilidad). Las conclusiones que surgen a partir del mismo parecen indicar que estos aspectos son de tipo multidimensional, y según el contexto socio-cultural pueden aumentar o disminuir (DAVIS, 1989; BLASCO; MELÉNDEZ, 2006).

En general se entiende que la edad, la educación, una inteligencia flexible son factores que pueden predecir el uso de tecnología. Asimismo es necesario tener en cuenta que los cambios físicos y cognitivos son factores de dificultad en el uso de las computadores (POUSADA; DE LA FUENTE, 2006), como ser visión más débil, hipoacusia o sordera, problemas de coordinación psicomotora y de concentración (HAWTHORN, 2000).

Por tanto un factor relevante a tener en cuenta en la facilidad del uso de las mismas son letras más grandes, sonidos diferenciados apropiados, y menos necesidad de movimientos del mouse. Si el adulto mayor se ha de adaptar a la computadora, la misma se ha de adaptar igualmente al adulto mayor... Sin duda un factor esencial es la motivación y el sentimiento asociado de confort, competencia y control de los procesos (CZAJA et al., 1998; POUSADA; DE LA FUENTE, 2006). Otros estudios insisten que la actitud hacia las nuevas tecnologías no es perjudicial en sí (FESTERVAND; MEINERT; VITELL, 1994); y en consecuencia algunos adultos mayores encuentran beneficio en la computadora (SAUNDERS, 2004); mientras que otros se sienten muy viejos para aprender sobre el tema (TURNER; TURNER; WALLE, 2007); especialmente con aprehensión por cometer errores (BIRDI; ZAPF, 1997; SAUNDERS, 2004).

Un factor sin duda decisivo es el tipo y calidad de instrucción que se recibe sobre el uso de las computadoras (PUIG, 2000; CALERO, 2003; ZAMARRÓN; TÁRRAGA; FERNÁNDEZ-BALLESTEROS, 2008; GARAMENDI et al., 2010). Lo que para las jóvenes generaciones parece ser una relación inmediata e instantánea, para los adultos mayores implica una inversión mayor de tiempo y esfuerzo (CHARNESS; BORITZ; SCHUMANN, 1992; CZAJA et al., 1989; ELIAS; ELIAS; ROBBINS; GAGE, 1987; GOMEZ et al., 1986).

Los adultos mayores cometen más errores, requieren de mayor ayuda y tienen puntuaciones más bajas en los entrenamientos de prueba (GOMEZ et al., 1986; ELIAS et al., 1987; CHARNESS; BORITZ; SCHUMANN, 1992). Sin embargo estos factores se pueden compensar con programas psicopedagógicos que se consideran ofrecen herramientas cognitivas adaptativas para las nuevas tecnologías a los adultos mayores (DAVICINO et al., 2009; BINOTTI et al., 2009). Por otro lado, otras investigaciones destacan que la capacidad de aprendizaje se mantiene aun en adultos mayores (YUNI; URBANO, 2005). Es imprescindible insistir, por tanto - como conclusión preliminar - que el vínculo 
con las tecnologías "avanzadas" ha de ser progresivo, tolerante y adaptado a la realidad cognitiva e intelectual del adulto mayor, a efectos de facilitar su incorporación a su vida cotidiana evitando que su presencia se vuelva perturbante o persecutoria (CERELLA; POON; WILLIAMS, 1980).

\subsection{Actitudes hacia las computadoras}

La mayoría de las investigaciones (GREEN; MCADAMS, 2003; LOGES; JUNG, 2001; MURDOCK, 2002; NEGROPONTE, 1995; WEINGARDT, 2000; FAULKNER, 2001; WILSON, 1973; DOCTOR, 1994; ROGERS; SHOEMAKER, 1971; WILSON, 1973; MADDEN; SAVAGE, 2000; EDGE, 1995; WOOLGAR, 1996) parecen indicar que no existe un usuario homogéneo frente al uso de las nuevas tecnologías. Al mismo tiempo parece ir prevaleciendo la tendencia de que los adultos mayores pueden sostener progresivamente una actitud positiva y menos ansiosa ante las computadoras (DYCK; SMITHER, 1994; TEMPLE; GAVILLET, 1990; SALOMON; PERKINS, 1996).

Por otro lado cuando el adulto mayor tuvo oportunidad de estar en contacto previo con tecnología o computadoras en su vida laboral, parece existir una correlación positiva que facilita el uso de las mismas (por ejemplo, el caso de ingenieros jubilados) (LOYD; GRESSARD, 1984). El aprendizaje que se realice es sin embargo también fundamental, aunque también aquí el tema es materia de debate. Mientras que la investigación de Gilroy y Desai (1986), encuentra una correlación positiva, Czaja et al. (1989) no encuentra diferencias fundamentales en la actitud hacia la computadora como resultado de procesos de entrenamiento.

Aunque existen pocos estudios sobre factores cognitivos en la adquisición de habilidades para la computación, los mismos parecen ser una influencia decisiva (MAYER; DYCK; VILBERG, 1986; SHUTE, 1991; VAN DER VEER, 1989). Especialmente se ha destacado la importancia de la habilidad espacial, específicamente para el uso del editor de texto (EGAN, 1988). Por otro lado la capacidad deductiva y de razonamiento, ha recibido poca atención y sin resultados concluyentes, especialmente en lo que hace a factores inductivos (GOMEZ et al., 1986).

Un factor que parece importante en el cambio en la actitud hacia las computadoras es el tiempo que se dedique a su aprendizaje, más allá de los contenidos en sí que se utilicen (CHARNESS; BORITZ; SCHUMANN, 1992; CZAJA et al., 1989; DYCK; SMITHER, 1994). Estas investigaciones parecen sugerir que no hay necesariamente un prejuicio "a priori" del adulto mayor hacia las computadoras, aunque no tengan suficiente experiencia con las mismas (HARRINGTON; MCELROY; MORROW, 1990; JACKSON; VOLLMER; 
STUURMAN, 1985; MARCOULIDES, 1988). Sin desmerecer los aspectos cognitivos mencionados, parece importante mencionar también el sostén que el adulto mayor recibe de sus redes de apoyo y especialmente familiares, como hijos o nietos (KLEIN, 2009), factores relevantes que son sin embargo no tenidos en cuenta en la bibliografía consultada (VAN DER WARDT; BANDELOW; HOGERVORST, 2012; CHUTTUR, 2009; EZER; FISK; ROGERS, 2009; KIBIK, 2009; OPPENAUER, 2009; GOLDMAN, 2007; BOUMA et al., 2007).

Un elemento relacionado y que es relevante para el uso de las nuevas tecnologías es la comunicación y los soportes sociales (MCMELLON; SCHIFFMAN, 2000; MORRELL et al., 2000; MANN et al., 2005). Otro elemento refiere a placer y entretenimiento (MCMELLON; SCHIFFMAN, 2000; WHITE et al.; WEATHERALL, 2000) con intereses por hobbies como la genealogía; información sobre salud (TAK; HONG, 2005; FLYNN; SMITH; FREESE; 2006; CAMPBELL, 2008; MACIAS; MCMILLAN, 2008); educación (DORIN, 2007); contacto con familia y amigos (THAYER; RAY, 2006; WHITE et al.; WEATHERALL, 2000; OPALINSKI, 2001; ALEXY, 2000) y aumento de la capacidad creativa (CAMPBELL, 2008; ROSENTHAL, 2008; BLAKE, 1998).

Hay que profundizar en este punto ya que muchas veces las computadoras presentan una oportunidad única para que los adultos mayores puedan establecer redes sociales que pueden aliviar la soledad y el aislamiento. La pérdida de parientes, amigos cercanos, o pérdida de conexiones sociales debido a los problemas de la edad y de paso del tiempo, son realidades dolorosas que el adulto mayor debe enfrentar. Por ende, forjar nuevas relaciones es frecuentemente uno de los desafíos que el adulto mayor debe enfrentar, para no caer en depresión y soledad (KLEIN, 2009; 2013).

Las computadoras e internet proveen un espacio donde se pueden encontrar temas de interés y otras personas con problemas similares (COULSON, 2000; LAWHORN et al., 1996; OGOZALEK, 1991; RYAN; HEAVEN, 1986). Alpass y Neville (2003) investigaron la relación entre soledad, saludos y depresión. Sin embargo un estudio de White et al. (2002) indica que estadísticamente no es significativo la reducción de estos factores con el uso de la computadora e internet.

En otros casos es importante recalcar que el uso de estas tecnologías sin duda proveen experiencias vitales reaseguradoras y exitosas (KNUFER, 1997; COLLIS, 1985, 1985B; MCMELLON; SCHIFFMAN, 2002; BECKERS; SCHMIDT, 2001; ROSENTHAL, 2008; VUORI; HOLMLUND-RYTKÖNEN, 2005; JUNCOS; PEREIRO; FACAL, 2006). La bibliografía más reciente parece confirmar estos datos (SANTANA, 2015; BILBAO-OSORIO; DUTTA; LANVIN, 2014).

Sin embargo esta afirmación - como conclusión preliminar - debe ser discutida ampliamente con mayor precisión y amerita un trabajo específico 
sobre el tema, pues esta realidad se aplica especialmente para los llamados países "desarrollados" (EUA, Europa). En los países llamados "en desarrollo" se verifican contextos de fuerte desigualdad en el acceso a las nuevas tecnologías y a bienes y servicios en general, como parte de profundos procesos de desigualdad económica, social y cultural (MARQUINE; SANTANA, 2016).

Pero aún en los países "en desarrollo" es necesario destacar la heterogeneidad en los procesos de incorporación a las nuevas tecnologías. El uso de las mismas parece depender de contextos familiares, emocionales, cognitivas y de la profesión que se tenía antes de la jubilación (GOMEZ et al., 1986). No se puede sino concluir que los estudios no son para nada concluyentes y demuestran que estamos aún ante un tema de debate inconcluso (DUKES; DISCENZA; COUGER, 1989; GILROY; DESAI, 1986; KOOHANG, 1989; MASSOUD, 1991; MORROW; PRELL; MCELROY, 1986).

\subsection{Resultados encontrados}

Un estudio general sobre los resultados encontrados parecen coincidir en algunos aspectos. Desde el punto cuantitativo, refiere a que de una u otra manera las nuevas tecnologías se han ido incorporando al mundo de los adultos mayores. De cualquier manera cualitativamente no se pueden ignorar la persistencia de desigualdades sociales y económicas las que atentan contra la difusión generalizada de estas nuevas tecnologías.

La expectativa de que solo los jóvenes serían usuarios de las nuevas tecnologías no se ha cumplido. Los adultos mayores progresivamente han sabido hacer uso positivo de las nuevas tecnologías, aunque posiblemente este aprendizaje lleva más tiempo y esfuerzo. Sin embargo, como se ha señalado, los nietos, especialmente los nietos adolescentes han jugado un papel fundamental en el aprendizaje de las nuevas tecnologías, factor que las investigaciones no han tenido suficientemente en cuenta (KLEIN, 2009).

Parece importante señalar también que si bien en algunos puntos las conclusiones coinciden en otros divergen. Especialmente, es importante enfatizar el debate sobre si el uso de las nuevas tecnologías incrementa o no la calidad de vida de los adultos mayores. Aquí las diferencias cualitativas son especialmente relevantes. Si las profesiones que los adultos mayores tenían antes de su retiro o jubilación, permiten una mejor predisposición hacia las nuevas tecnología, parece haber un incremento en la calidad de vida. En otros casos, las nuevas tecnologías no parecen influir decisivamente en uno u otro sentido (MARQUINE; SANTANA, 2014). 
Las observaciones señaladas en los apartados anteriores merecen algunas reflexiones críticas. Una de ellas es que en los comienzos de la difusión de las nuevas tecnologías se depositó en ella una confianza excesivamente optimista o mesiánica para mejorar o asentar la calidad de vida de los adultos mayores, lo que no necesariamente se ha verificado (MARQUINE; SANTANA, 2016).

El transcurso del tiempo ha demostrado que la calidad de vida de los adultos mayores no depende solo o mágicamente de tecnologías, que muchas veces son parte de un dispositivo de una sociedad consumista, sino que es indisociable de políticas públicas eficaces que los autores han ignorado en sus investigaciones (KLEIN, 2016). Tampoco se ha señalado claramente que la difusión de las nuevas tecnologías no es homogéneo sino heterogéneo pues depende de contextos sociales, económicas y culturales que atraviesan el mundo de hoy, donde productos, ingresos y oportunidades de vida son marcados por profundas y radicales desigualdades (SANTANA, 2015).

Por otra parte, otro de los indicadores que interesaba indagar - según se señala en la Metodologia - era si las tecnologías incidían o no en los procesos de ciudadanía. No se ha encontrado material significativo en tal sentido, por más que todo hace indicar que los procesos de envejecimiento poblacional incrementarán cada vez más el poder político de los adultos mayores (KLEIN; AVILA EGGLETON, 2011). Asimismo es necesario insistir en los estudios de género en relación a las nuevas tecnologías. Si bien existen estudios importantes (VAN DER WARDT; BANDELOW; HOGERVORST, 2012; CHUTTUR, 2009; EZER; FISK; ROGERS, 2009) que han sido ya destacados, se hace necesario verificar si el paso del tiempo corrobora o no los mismos en el sentido de una mayor dificultad femenina en el acceso a las nuevas tecnologías.

Por último es necesario señalar que muchas veces la frontera del acceso a las nuevas tecnologías como decisión consciente del adulto mayor o como coacción, dentro de los que se consensuan como "políticas de salud", desde un dispositivo que impone que los adultos mayores han de estar ocupados y productivos, no es para nada clara. En tal sentido, las tecnologías han sido usadas muchas veces como parte de una estructura disciplinante biopolítica (FOUCAULT, 2004). 
El presente manuscrito intenta presentar el estado de la cuestión en relación al uso de las tecnologías en los adultos mayores.

Los descriptores utilizados se enfocan en:

-si la misma ha sido un obstáculo o no en la calidad de vida de los mismos;

-si se ha incorporado exitosamente o no a sus actividades cotidianas;

-si la tecnología ha incidido en procesos de agenciamiento y ciudadanización.

La metodología utilizada fue una revisión bibliográfica extensa y profunda sobre el tema teniendo en cuenta la perspectiva de diferentes autores, pero también investigaciones que conciernen a diferentes países, tratando de tener en cuenta una perspectiva lo más amplia posible. La base de datos utilizada fue Latindex, Dialnet, Red de Bibliotecas Universitarias (REBIUN) CIRC 2012 y principalmente la base de datos de la Universidad de Oxford y del Oxford Institute of Population Ageing: la Oxford University Central Library (The Bodleian Library) y la Social Sciences Library.

La base de datos revisada trató de ser lo más exhaustiva posible: comienza en los años 70 y llega como año de corte hasta el año 2016. Se hace notar que el mayor auge de investigaciones se realiza a comienzos del siglo XX. Los idiomas de la base de datos fueron principalmente inglés, español y portugués y las palabras clave o descriptores utilizados fueron aquellos que permitieran un estudio del tema incorporando elementos que dieran cuenta fehacientemente de su complejidad y multiplicidad de variables en juego.

Por ende, el criterio de inclusión fue incorporar aquellos materiales que proporcionan una perspectiva los más interdisciplinaria posible, recogiendo información de la sociología, la antropología, la psicología y la informática entre otras. El criterio de exclusión fueron trabajos que no aportan criterios originales o con conclusiones que no aportan al debate del tema.

\section{Consideraciones finales}

La revisión presentada indica que a pesar del gran número de investigaciones realizadas, muchas de las predicciones realizadas en el sentido de una resistencia del adulto mayor ante las nuevas tecnologías no se ha cumplido. La afirmación de que las nuevas tecnologías pertenecen al mundo de los jóvenes (BUCUR; RENOLD; HENKE, 1999) es incorrecta e inexacta.

Quizás un error es restringir el mundo de las nuevas tecnologías como propio del mundo joven, con lo que los adultos mayores quedarìan en una situación 
de desventaja. En otras palabras el mundo de la tecnología llegaría "tarde" al mundo de los adultos mayores de forma impositiva y quizás persecutoria.

La bibliografía de los últimos cinco años (SANTANA, 2015; MARQUINE; SANTANA, 2014, 2016; BILBAO-OSORIO; DUTTA; LANVIN, 2014) indican que el acceso a las nuevas tecnologías se considera imprescindible para una mejor adaptación e integración del adulto mayor a su contexto familiar, cultural, social. Dato que se va tornando irreversible indicando nuevas pautas culturales y sociales que se han vuelto imprescindibles e imposibles de ignorar.

Aunque originariamente se pensaba que el adulto mayor sería indiferente o sentiría ansiedad antes las nuevas tecnologías, esto no se ha comprobado (SANTANA, 2015). Quizás podamos decir que el adulto mayor no está ni en contra ni a favor de la tecnología. Sin duda que la tecnología es percibida por éstos como facilitadores de información, logros en el manejo de su salud, creación de redes y soportes sociales. Pero no garantiza per se calidad de vida, la que depende entre otros factores, de políticas sociales eficientes y redistributivas.

Podemos enunciar la hipótesis de que lo destacable no radica en la computadora en sí, ni tampoco en el abandono de formas tradicionales de comunicación y de transmisión que se suponían formaban parte de la identidad del adulto mayor, sino de un factor de capacidad de cambio y adaptación a situaciones nuevas que los adultos mayores han sabido manejar de forma eficaz.

Todo parece indicar que el adulto mayor disfruta y se complace de los beneficios de las nuevas tecnologías a las que se adapta - aunque a veces con dificultades - sin problemas. Sin embargo ya se señaló que tampoco se puede desconocer cierta "coacción" de ciertos paradigmas predominantes de salud en el uso de computadores, como parte de un dispositivo disciplinante y de un estereotipo de que el adulto mayor debe ser "moderno", "adaptado" y siempre ocupado en actividades "productivas".

\section{THE GROWING USE OF NEW TECHNOLOGIES AMONG OLD PEOPLE}

\section{abstract}

As computers become an increasingly integral part of the lives of older adults, the study of computer use by older adults is becoming an increasingly relevant field of study. The aim of the study is a bibliographic review to better understand the relationship of new technologies with the population of older adults. One of the consequences that appears is that in order to encourage and facilitate the 

and professional benefit, it is necessary to understand the implication of this new technologies and cultural traditions. The methodology used is bibliographical review with emphasized both in the evolution of academic perspectives on the subject and the results obtained between the years 1970-2016. It should be noted that all the researches consulted insist on the increasingly important role of the new technology is in the life of the elderly, and that far from assuming that ageing people would have a rejection to the same, is possible to perceptive an operative and surprising use of the new technologies.

\section{keywords}

Information Society. New Technologies. Ageing.

\section{referencias}

ADLER, Robert. Older adults and computers. 1996. Disponible en: <http://www.seniornet. Org/php/default.php?PagelD=5476yVersion=0yFont=0>. Acceso en: 22 enero 2015.

ALEXY, Ernest. Computers and caregiving: reaching out and redesigning interventions for homebound older adults and caregivers. Holistic Nursing Practice, USA, v. 14, n. 4, p. 60-66, 2000.

ALPASS, Fiona; NEVILLE, Stephen. Loneliness, health, and depression in older males. Aging and Mental Health, v. 7. n. 3, p. 212-217, 2003.

AMERICAN ASSOCIATION OF RETIRED PERSONS (AARP). Understanding senior housing into the next century: survey of consumer preferences, concerns, and needs. Washington, D.C.: American Association of Retired Persons, 1996. 2008. Healthy@ home. Washington, DC: American Association of Retired Persons,

BALTES, Margret et al. Everyday competence in old and very old age: theoretical considerations and empirical findings. In: BALTES, P.; MAYER, K. (Org.). The Berlin aging study: aging from 70 to 100. Cambridge: Cambridge University Press, 1999. p. 384-402.

BECKERS, John; SCHMIDT, Henk. The structure of computer anxiety: a six-factor model. Computers in Human Behavior, v. 18, n. 17, p. 35-49, jul. 2001.

BERNARD, Miriam; PHILLIPS, Judith. The challenge of ageing in tomorrow's Britain. Ageing and Society, v. 43, n. 20, p. 33-54, 2000.

BILBAO-OSORIO, Beñat; DUTTA, Soumitra; LANVIN, Bruno (Ed.). The Global Information Technology Report 2014: Rewards and Risks of Big Data. World Economic Forum: Genebra, 2014. Disponible en: <http://www.weforum.Org/docs/WEF_GloballnformationTechnology_Report_2014.pdf>.Acceso en: 18 sept. 2016.

BINOTTI, Pedro et al. Funciones ejecutivas y aprendizaje en el envejecimiento normal. Estimulación cognitiva desde una mirada psicopedagógica. Revista Chilena de Neuropsicología, Santiago, v. 4, n. 2, p. 119-126, 2009. 
BIRDI, Kamaljit; ZAPF, Dieter. Age differences in reactions to errors in computer based Work. Behaviour \& Information Technology, v. 16, n. 6, p. 309-319, 1997.

BLASCO, Sonia; MELÉNDEZ, Juan. Cambios en la memoria asociados al envejecimiento. Geriatrika: Revista Iberoamericana de Gerontología y Geriatría, v. 23, n. 22 p. $179-185,2006$

BOUMA, Herman et al. Gerontechnology in perspective. Gerontechnology, v. 6, n. 4, p. 190-216, 2007.

BRAYFIELD, Celia. Rise of the silver surfers. The Times, v. 11, n. 37, p. 125-167, 2000

BUCUR, Alexander; RENOLD, Carl; HENKE, Maria. How do older netcitizens compare with their younger counterparts? Cyberpsychology \& Behavior, v. 2, n. 6, p. 505-513, 1999

BURDICK, Donald. Digital divide or tool for understanding and collaboration: computers and intergenerational relationships. In: ANNUAL SCIENTIFIC MEETING OF THE GERONTOLOGICAL SOCIETY OF AMERICAS, 54., 2001, Chicago. Anais.. Washington: Gerontological Society of America, 2001.

CALERO, Martín. La utilidad de los programas de intervención cognitiva en personas mayores. Geriatrika: Revista Iberoamericana de Gerontología y Geriatría, v. 38, n. 6, p. 305-307, 2003.

CAMPBELL, Robert James. Meeting seniors' information needs: using computer Technology. Home Health Care Management and Practice, v. 20, n. 4, p. 328-335, 2008.

CERDA, Aida. Alfabetización digital en el Adulto Mayor: ¿En el camino de la inclusión social? Santiago: Universidad de Chile, 2005. Disponible en: <http://www. archivochile. com/tesis/09_tedulit/09tedulit0016.pdf>. Acceso en: 16 mayo 2015.

CERELLA, John; POON, Leonard; WILLIAMS, Diane. Age and the complexity hypothesis. In: POON, Leonard W. (Org.). Aging in the 1980s. Washington, D. C.: American Psychological Association, 1980. p. 332-340.

CODY, Michael et al. Silver surfers: training and evaluating internet use among older adult learners. Communication Education, Raleigh, v. 11, n. 48, p. 269-286, 1999.

COLLIS, Betty. Psychosocial implications of sex differences in attitudes towards computers: result of a survey. International Journal of Women's Studies, Massachusetts, v. 8, n. 3, p. 207-213, 1985.

Sex-related differences in attitudes toward computers: implications for counselors. The School Counselor, Alexandria, VA, v. 34, n. 12, p. 120-130, 1985b.

COULSON, Ingrid. Introduction: technological challenges for gerontologists in the 21st century. Educational Gerontology, v. 26, n. 4, p. 307-316, 2000.

COPPS, Alex. Gold mine awaits in the silver-surfer market. The Times, v. 10, n. 4 p. 2-12, 2000 .

CHARNESS, Neil; BORITZ, Gayla; SCHUMANN, Cynthia. Training older adults in word processing: Effects of age, training technique, and computer anxiety. International Journal of Technology \& Aging, v. 12, n. 5, p. 79-106, 1992.

CHUTTUR, Mohammad. Overview of the Technology acceptance model: origins, developments and future directions. Sprouts, v. 9, n. 37, p. 9-37, 2009.

CZAJA, Sara et al. Age related differences in learning to use a text-editing system. Behaviour and Information Technology, v. 14, n. 8, p. 309-319, 1989.

DAVICINO, Noelia et al. El rol psicopedagógico en la Estimulación Cognitiva de pacientes con demencia tipo Alzheimer. Revista Chilena de Neuropsicología, Santiago, v. 4 n. 1, p. 6-11, 2009.

DAVIS, Fred. Perceived usefulness, perceived ease of use and user acceptance of information technology. MIS Quarterly, Minnesota, v. 15, n. 13, p. 319-339, 1989. 
DORIN, Michelle. Online education of older adults and its relation to life satisfaction. Educational Gerontology, v. 33, n. 2, p. 127-143, 2007.

DUKES, Richard; DISCENZA, Richard; COUGER, J. Daniel. Convergent validity of four computer anxiety scales. Educational and Psychological Measurement, v. 12, n. 49, p. $151-158,1989$.

DYCK, Jennifer; SMITHER, Janan. Age differences in computer anxiety: the role of computer experience, gender and education. Journal of Educational Computing Research, v. 19, n. 10, p. 231-240, 1994

EDGE, David. The social shaping of technology. In: HEAP, N. et al. (Org.). Information Technology and society. London: Sage, 1995. p. 14-32.

EGAN, David. Individual differences in human-computer interaction. Psychology and Aging, v. 2, n. 12, p. 340-348, 1988.

EZER, Neta; FISK, Arthur; ROGERS, Wendy. Attitudinal and intentional acceptance of domestic robots by younger and older adults. Universal Access in Human-Computer Interaction. Intelligent and Ubiquitous Interaction Environments. Lecture Notes in Computer Science, v. 56, n. 15, p. 39-48, 2009.

FAULKNER, Wendy. The technology question in feminism: a view from feminist technology studies. Women's Studies International Forum, v. 24, n. 1, p. 79-95, 2001.

FESTERVAND, Troy; MEINERT, David; VITELL, Scott. Older adults' attitudes toward and adoption of personal computers and computer-based lifestyle assistance. Journal of Applied Business Research, v. 10, n. 2, p. 13-22, 1994.

FIELD, Thomas et al. The costs associated with adverse drug events among older adults in the ambulatory setting. Medical Care, v. 43, n. 12, p. 1171-1176, 2005.

FLYNN, Kathryn; SMITH, Maureen; FREESE, Jeremy. When do older adults turn to the internet for health information? Findings from the Wisconsin longitudinal study. Journal of General Internal Medicine, v. 21, n. 12, p. 1295-1301, 2006.

FOUCAULT, Michael. La tecnología política de los individuos. Rio de Janeiro: Forense Universitária, 2004. p. 302-389. (Coleção Ditos \& Escritos V).

GARAMENDI, Franciso et al. Programa de entrenamiento cognitivo en adultos mayores. Revista Mexicana de Medicina Física y Rehabilitación, Ciudad de México, v. 34, n. 22, p. 26-31, 2010 .

GILROY, Faith; DESSAI, Harsha. Computer anxiety: sex, race and age. International Journal of Man-Machine Studies, v. 12, n. 25, p. 711-719, 1986

GOMEZ, Luis et al. Learning to use a text editor: some learner characteristics that predtic success. Human-Computer Interaction, v. 22, n. 2, p. 1-23, 1986.

GREEN, R.; MCADAMS, Dan. The souls of digital black folks: a narrative study of digitally fluent African-Americans. Paper presented to the American Educational Research Association Annuals Conference, Chicago, 2003.

HANLEY, Paul. The numbers game: older people and the media. Londres: Independent Television Commission, 2002.

HARRINGTON, Kermith; MCELROY, James; MORROW, Paula. Computer anxiety and computer based training: a laboratory experiment. Journal of Educational Computing Research, v. 11, n. 6, p. 343-358, 1990.

HART, Traci; HALCOMB, Charles; CHAPARRO, Barbara. Evaluating websites for older adults: Adherence to senior-friendly guidelines and end-user performance. Behaviour \& Information Technology, v. 27, n. 3, p. 191-199, 2008

HAWTHORN, Daniel. Possible implications of aging for interface designers. Interacting with Computers, Birmingham, v. 12, n. 5, p. 507-528, 2000 
IRIZARRY, Carol; DOWNING, A. Computers enhancing the lives of older people. Australian Journal on Ageing, v. 16, n. 4, p. 161-165, 1997.

JACKSON, Lorraine; VOLLMER, Lorraine; STUURMAN, John. Effects of attitude and task complexity on microcomputer text-editing. Journal of Computer-Based Instruction, Ohio, v. 14, n. 12, p. 111-115, 1985.

JARA, Madrigal. La estimulación cognitiva en personas adultas mayores. Revista Cúpula, v. 22, n. 2, p. 4-14, 2008.

KLEIN, Alejandro; ÁVILA-EGGLETON, Marcela. De los modelos de ciudadanía sólida a un modelo de ciudadanía alternante-intermitente. Revista Publicatio, Ponta Grossa, v. 19, n. 1, p. 85-94, 2011.

KLEIN, Alejandro. Nuevas formas de relacionamiento abuelos-nietos adolescentes desde los cambios demográficos-sociales actuales. Psicología Revista, São Paulo, v. 12, n. 18, p. $1-25,2009$.

Promesa extinguida o promesa en estado de fluido: continuidades y discontinuidades de los adultos mayores hoy. Revista Psicologia \& Sociedade, Minas Gerais, v. 25, n. 1, p. 213-219, 2013.

El drama de ser pobre, viejo y latinoamericano. El caso de México. Larna Newsletter, v. 20, n. 6, p. 5-17, 2016.

KNUFER, Nancy Nelson. Gender by design. Educational Technology, v. 37, n. 2 , p. 31-37, 1997.

KOOHANG, Alex. A study of attitudes toward computers: anxiety, confidence, liking, and perception of usefulness. Journal of Research on Computing in Education, v. 32, n. 22, p. 137-150, 1989.

KUBIK, Sara. Motivations for cell phone use by older Americans. Gerontechnology, v. 8, n. 3, p. 150-164, 2009

LAWHORN, Thomas et al. Seniors adults and computers in the 1900's. Educational Gerontology, v. 22, n. 2, p. 193-201, 1996.

LEESON, George. Demography, Politics and Policy in Europe. In: LUDLOW, Peter (Org.). Setting EU Priorities. Ponte de Lima: The European Strategy Forum, 2009. p. 102-124.

The demographics of population ageing in Latin America, the Caribbean and the Iberian Peninsula, 1950-2050. In: MONTES DE OCA, Verónica (Org.). La agenda del Envejecimiento y las Políticas Públicas Hoy. Mexico: Instituto de Investigaciones Sociales, p. 53-74, 2013.

LOGES, William; JUNG, Joo-Young. Exploring the digital divide: internet connectedness and age. Communication Research, v. 28, n. 4, p. 536-562, 2001.

MACIAS, Wendy; MCMILLAN, Sally. The return of the house call: the role of internet based interactivity in bringing health information home to older adults. Health Communications, v. 23, n. 1, p. 34-44, 2008.

MADDEN, Gary; SAVAGE, Scott. Some economic and social aspects of residential Internet use in Australia. Journal of Media Economics, v. 13, n. 3, p. 171-185, 2000

MANN, William et al. Computer use by middle-aged and older adults with disabilities, Technology and Disability, v. 17, n. 1, p. 1-9, 2005

MARCOULIDES, George. The relationship between computer anxiety and computer Achievement. Journal of Educational Computing Research, v. 19, n. 4, p. 151-158, 1988.

MARQUINE, Taiuani Raymundo; SANTANA, Cesar C. Factors Influencing the Acceptance of Technology by Older People: how the elderly in Brazil feel about using electronics. Consumer Electronics Magazine, v. 3, n. 4, p. 63-68, 2014. 

v. 18, n. 55 , p. $12-25,2016$

MASSOUD, Samia. Computer attitudes and computer knowledge of adult students. Journal of Educational Computing Research, v. 12, n. 7, p. 269-291, 1991.

MAYER, Richard; DYCK, Jennifer; VILBERG, William. Learning to program and learning to think: what's the connection? Communications of the ACM, v. 26, n. 29, p. 605-610, 1986.

MCMELLON, Charles; SCHIFFMAN, Leon. Cyber senior mobility: why some older consumers may be adopting the Internet. Advances in Consumer Research, v. 27, n. 1, p. 139-144, 2000.

Cyber senior empowerment: how some older individuals are taking control of their lives. Journal of Applied Gerontology, v. 21, n. 2, p. 157-175, 2002.

MELENHORST, Anne-Sophie; ROGERS, Wendy Anne; BOUWHUIS, D.G. Older adults' motivated choice for technological innovation: Evidence for benefit-driven selectivity. Psychology and Aging, v. 21, n. 1, p. 190-195, 2006.

MORRELL, Robert et al. A survey of World Wide Web use in middle-aged and older adults. Human Factors, v. 42, n. 2, p. 175-182, 2000

MORROW, Paula; PRELL, E.R.; MCELROY, James. Attitudinal and behavioral correlates of computer anxiety. Psychological Reports, v. 15, n. 59, p. 1199-1204, 1986.

MURDOCK, Graham. Tackling the digital divide: evidence and intervention. Coventry: British Educational Communications and Technology Agency, 2002. p. 111-128.

NEGROPONTE, Nicholas. Being digital. Londres: Coronet, 1995.

OPALINSKI, Laura. Older adults and the digital divide: assessing results of a web based Survey. Journal of Technology in Human Services, v. 18, n. 3, p. 203-221, 2001.

OPPENAUER, Claudia. Motivation and needs for technology use in old age. Gerontechnology, v. 8, n. 2, p. 82-87, 2009 .

POUSADA, Modesta; DE LA FUENTE, Javier. Memoria y Atención. In: TRIADÓ, Carlos; VILLAR, Francisco (Org.). Psicología de la vejez. Madrid: Alianza Editorial, 2006. p. 300-326.

PUIG, Alemán. Un instrumento eficaz para prevenir el deterioro cognitivo de los ancianos institucionalizados: El Programa de Psicoestimulación Preventiva (PPP). Revista Multidisciplinaria de Gerontología, v. 10, n. 3, p. 146-151, 2000.

ROGERS, Everett; SHOEMAKER, F. Floyd. Communication of innovations. New York: Free Press, 1971

ROSENTHAL, Rita. Older computer-literate women: their motivations, obstacles, and paths to success. Educational Gerontology, v. 34, n. 7, p. 610-626, 2008.

RYAN, Ellen Brouchard; HEAVEN, Roberta. Promoting vitality among older adults with computers. Activities, Adaptation \& Aging, v. 8, n. 1, p. 15-30, 1986.

SALOMON, Gavriel; PERIKINS, David. Learning in wonderland: What computers really offer education. In: KERR, Susan (Org.). Technology and the future of education. Chicago: University of Chicago Press, p. 201-245, 1996

SANTANA, Carla da Silva; LEESON, George. Elderly Users' Perspective on the Use of Technology in Daily Life: A Comparative Study of a sample in the UK and Brazil. Inteligencia Artificial, v. 18, n. 55, p. 35-49, 2015.

SAUNDERS, Edward. Maximizing computer use among the elderly in rural senior Centers. Educational Gerontology, v. 30, n. 7, p. 573-585, 2004.

SHUTE, Valerie. Who is likely to acquire programming skills? Journal of Educational Computing Research, v. 8, n. 7, p. 1-24, 1991 
TAK, Sunghee; HONG, Song Hee. Use of the internet for health information by older adults with arthritis. Orthopaedic Nursing, v. 24, n. 2, p. 134-138, 2005.

TEMPLE, Lori; GAVILLET, Margaret. The development of computer confidence in seniors: an assessment of changes in computer anxiety and computer literacy. Activities, Adaptation y Aging, v. 14, n. 3, p. 63-76, 1990

TEO, Thompson. Demographic and motivation variables associated with Internet usage activities. Internet Research: Electronic Networking Applications and Policy, v. 11, n. 2, p. 125-137, 2000.

TURNER, Phil; TURNER, Susan; WALLE, Guy Van de. How older people account for their experiences with interactive technology. Behaviour and Information Technology, v. 26, n. 4, p. 287-296, 2007.

UNITED NATIONS. World Population Prospects: The 2008 Revision, Highlights. New York: United Nations, 2009. Disponible en: <http://www.un.org/esa/population/publications/wpp2008/wpp2008_highlights.pdf>. Acceso en: 16 sept. 2016.

US CENSUS BUREAU. The 65 years and over population. 2000. Disponible en: <http:// www.census.gov/prod/2001pubs/c2kbr01-10.pdf>. Acceso en: 5 jun. 2015.

Use of a computer at home, school, or work and the Internet at any location for people 18 years and over, by selected characteristics. Table 5B. 2003. Disponible en: <http://www.census.gov/population/socdemo/computer/2003/tab05B.xls>. Acceso en: 10 jul. 2015.

VAN DER WARDT, Veronika; BANDELOW, Stephan; HOGERVORST, Eef. The relationship between cognitive abilities, well-being and use of new technologies in older people. Gerontechnology, v. 10, n. 4, p. 187-207, 2012.

VAN DER VEER, Gerrit. Individual differences and the user interface. Ergonomics, v. 14 n. 32, p. 1431-1449, 1989 .

VUORI, Satu; HOLMLUND-RYTKÖNEN, Maria. 55+ people as internet users. Marketing Intelligence \& Planning, v. 23, n. 1, p. 58-76, 2005.

WEINGARDT, Kenneth. Viewing ambivalence from a sociological perspective: implications for psychotherapists. Psychotherapy, v. 37, n. 4, p. 298-306, 2000.

WHITE, Henry et al. Surfing the net in later life: a review of the literature and pilot study of computer use and quality of life. Journal of Applied Gerontology, v. 18, n. 3, p. 358-378, 1999.

WHITE, Henry et al. A randomized controlled trial of the psychosocial impact of providing Internet training and access to older adults. Aging and Mental Health, v. 6 , n. 3, p. 213-222, 2002.

WILSON, Patrick. Situational relevance. Information Storage and Retrieval, v. 10, n. 9 p. 457-471, 1973.

WILLS, Martin. Bridging the digital divide. Adults Learning, v. 23, n. 24, p. 10-11, 1999.

WOOLGAR, Steve. Technologies as cultural artefacts. In: DUTTON, Walter (Org.). Information and communication technologies. Oxford: Oxford University Press, p. 87-102, 1996.

YUNI, José Alberto; URBANO, Claudio Ariel. Educación de adultos mayores: Teoría, investigación e Intervenciones. Buenos Aires: Brujas, 2005.

ZAMARRÓN, María; TÁGARRA, Lluís; FERNÁNDEZ-BALLESTEROS, Rocío. Plasticidad cognitiva en personas con la enfermedad de Alzheimer que reciben programas de estimulación cognitiva. Psicothema, v. 20, n. 3, p. 432-437, 2008. 
Data de submissão: 23/01/2016

Data de aprovação: 19/12/2017 